\title{
Robust Watermarking based on Subsampling and Nonnegative Matrix Factorization*
}

\author{
Wei LU ${ }^{1,2}$, Hongtao LU ${ }^{2}$ \\ ${ }^{1}$ Department of Computer Science and \\ Guangdong Key Laboratory of Information Security Technology \\ Sun Yat-sen University, Guangzhou 510275, China \\ ${ }^{2}$ Department of Computer Science and Engineering \\ Shanghai Jiao Tong University, Shanghai 200240, China \\ e-mail:luwei3@mail.sysu.edu.cn,lu-ht@cs.sjtu.edu.cn
}

Received: August 2007; accepted: May 2008

\begin{abstract}
This paper presents a novel robust digital image watermarking scheme using subsampling and nonnegative matrix factorization. Firstly, subsampling is used to construct a subimage sequence. Then, based on the column similarity of the subimage sequence, nonnegative matrix factorization (NMF) is applied to decompose the sequence. A Gaussian pseudo-random watermark sequence is embedded in the factorized decomposition coefficients. Because of the high similarity of subimages and meaningful factorization for NMF, the proposed scheme can achieve good robustness, especially to common permutation attacks. Numerical experiment results demonstrate the good performance of the proposed scheme.
\end{abstract}

Keywords: watermarking, nonnegative matrix factorization, DWT.

\section{Introduction}

In the past two decades, digital watermarking technology has been devoted significantly and applied in digital right protection and authentications widely (Chang and Tseng, 2004; Hassanien, 2007; Chang and Chang, 2007). On one hand, many different approaches are introduced into digital watermarking, such as signal processing, pattern recognition, communication theory etc., which have improved the performance of watermarking. On the other hand, most of the current watermarking schemes are still not applicable in practice, since the performance of these watermarking algorithms are still far from practical application, especially for robustness, which is the most important design criteria.

Recently, a new signal decomposition method is proposed as nonnegative matrix factorization (NMF) (Lee and Seung, 1999; Lee and Seung, 2001), which decomposes a nonnegative matrix into two physically meaningful nonnegative matrices, and has been

*This work was supported by NSFC (No. 60803136, No. 60573033), the Scientific Research Foundation for the Young Teachers in Sun Yat-sen University, and Program for New Century Excellent Talents in Uniersuty (No. NCET-05-0397). 
successfully applied in many signal analysis domains (Berrya et al., 2006), such as frontal face verification (Zafeiriou et al., 2006), blind signal separation (Cichocki et al., 2006), image classification (Guillamet et al., 2003). To our current knowledge, there are only a few applications for NMF in digital watermarking (Ghaderpanah and Hamza, 2006). In this paper, we propose a novel digital image watermarking scheme using NMF in subsampling domain. Through embedding a pseudo-random sequence in the NMF coefficient matrix, the proposed scheme can resist many signal attacks and distortions, and thus achieves strong robustness.

The rest parts of this paper are organized as follows. In Section 2, we introduce the subsampling technique. In Section 3, we introduce NMF algorithm briefly. In Section 4, the prosed watermarking scheme is described in detail. Then, the experimental results are shown in Section 5. Finally, conclusions are given in Section 6.

\section{Subsampling}

Subsampling is a common method in signal processing and analysis. For a digital image $I$ with size $M \times N$, subsampling decomposes it into $m n$ subimages of size $M / m \times N / n$, where $m$ and $n$ are the subsampling intervals on the direction of column and row, which can be described as follows:

$$
\begin{aligned}
& I_{1}(i, j)=I(m *(i-1)+1, n *(j-1)+1), \\
& I_{2}(i, j)=I(m *(i-1)+1, n *(j-1)+2), \\
& I_{3}(i, j)=I(m *(i-1)+1, n *(j-1)+3), \\
& \quad \ldots \ldots \\
& I_{n+1}(i, j)=I(m *(i-1)+2, n *(j-1)+1), \\
& I_{n+2}(i, j)=I(m *(i-1)+2, n *(j-1)+2), \\
& \quad \ldots \ldots \\
& I_{m n}(i, j)=I(m * i, n * j),
\end{aligned}
$$

where $I_{1}, I_{2}, \cdots, I_{m n}$ denote the subimages. Generally, there are high similarity among these submiages, i.e., the correlation coefficients between any two subimages are approximately equal to $1, \rho\left(I_{i}, I_{j}\right) \approx 1, i, j=1,2, \cdots, m n$. Fig. 1 shows some subimage examples, we can see that these subimages are highly similar. Based on this distinct character, subsampling has been used in watermarking (Chu, 2003; Lu et al., 2006). In (Chu, 2003), a subsampling based watermarking is proposed, which can achieve better robustness than Cox's scheme (Cox et al., 1997). However, there is a serious weakness for subsampling technique in this scheme. We have proposed a permutation attack to it and defeat the scheme simply and effectively (Lu et al., 2005). Then, a more robust anti-permutation watermarking was developed in (Lo et al., 2007). 


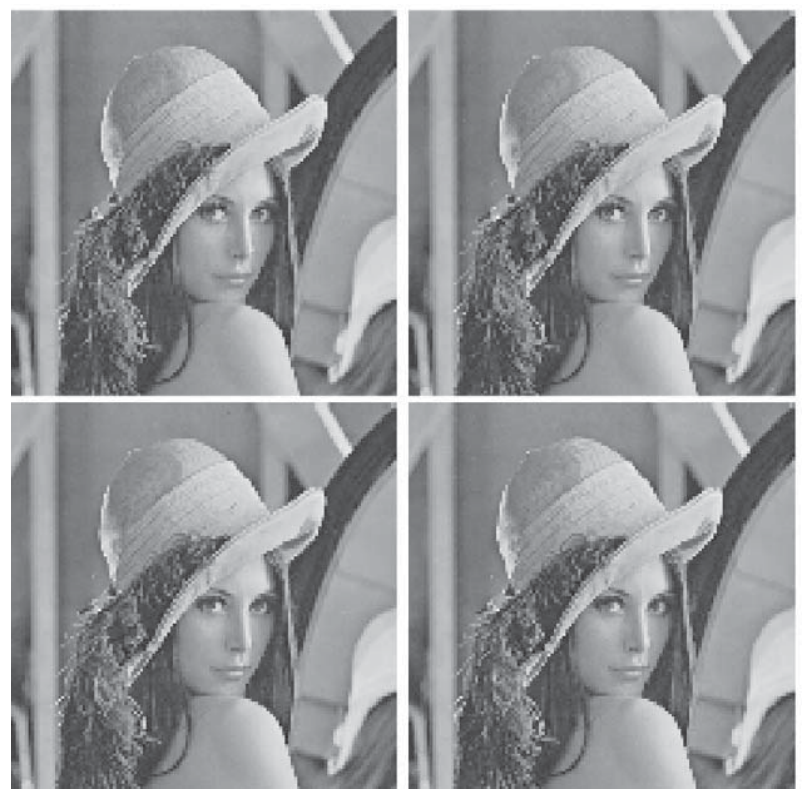

Fig. 1. The first four subimages from Lenna $(512 \times 512)$, where the subsampling parameter $m, n=4$.

\section{Nonnegative Matrix Factorization}

Nonnegative matrix factorization (NMF) is developed as a matrix factorization technique, which decomposes nonnegative matrices into physically meaningful data in two dimensional signal analysis, and has been used for image representation, document analysis and clustering for its parts-based representation property. NMF results in a reduced representation of the original data. Thus, NMF can also be a feature extraction or a dimensionality reduction technique. A formal description of nonnegative matrix factorization can be described as follows. Given a nonnegative matrix $I \in \mathbb{R}^{m \times n}$ and a positive integer $p<\min (m, n)$, NMF aims to find nonnegative matrix $W \in \mathbb{R}^{m \times p}$ and $H \in \mathbb{R}^{p \times n}$ to minimize the function

$$
f(W, H)=\frac{1}{2}\|I-W H\|_{F}^{2} .
$$

The product $W H$ is called a NMF of $I$, where $W$ is the normalized factor vectors by columns, $H$ is the encoding vectors. In other words, NMF decomposes a nonnegative matrix as follows:

$$
I \approx W H
$$

Here, we briefly describe the multiplicative iteration algorithm of NMF proposed in (Lee and Seung, 1999). Firstly, an objective function is selected based on the Poisson 
likelihood as follows:

$$
D(I, W H)=\sum_{i=1}^{m} \sum_{j=1}^{n}\left(I_{i j} \ln \frac{I_{i j}}{(W H)_{i j}}-I_{i j}+(W H)_{i j}\right)
$$

which, then, is simplified through some eliminiation of pure date terms, and we obtain

$$
D(I, W H)=\sum_{i=1}^{m} \sum_{j=1}^{n}\left(\sum_{k=1}^{p} W_{i k} H_{k j}-I_{i j} \ln \sum_{k=1}^{p} W_{i k} H_{k j}\right) .
$$

Taking the derivative with respect to $H$, we have

$$
\frac{\partial}{\partial H_{a b}} D(I, W H)=\sum_{i=1}^{p} W_{i a}-\sum_{i=1}^{p} \frac{I_{i b} W_{i a}}{\sum_{k=1}^{n} W_{i k} H_{k b}} .
$$

The gradient algorithm then states:

$$
\begin{aligned}
& H_{a b} \leftarrow H_{a b}-\eta_{a b} \frac{\partial}{\partial H_{a b}} D(I, W H), \\
& H_{a b} \leftarrow H_{a b}-\eta_{a b}\left[\sum_{i=1}^{p} \frac{I_{i b} W_{i a}}{\sum_{k=1}^{q} W_{i k} H_{k b}}-\sum_{i=1}^{p} W_{i a}\right]
\end{aligned}
$$

for some step size $\eta_{a b}$. Forcing

$$
\eta_{a b}=\frac{H_{a b}}{\sum_{i=1}^{p} W_{i a}}
$$

gives the multiplicative rule:

$$
H_{a b} \leftarrow H_{a b} \frac{\sum_{i=1}^{p}\left(W_{i a} I_{i b}\right) / \sum_{k=1}^{q} W_{i k} H_{k b}}{\sum_{i=1}^{p} W_{i a}} .
$$

Taking the derivative with respect to $W$ gives

$$
\frac{\partial}{\partial W_{c d}} D(I, W H)=\sum_{j=1}^{n} H_{d j}-\sum_{j=1}^{n} \frac{I_{c j} H_{d j}}{\sum_{k=1}^{q} W_{c k} H_{k j}} .
$$

The gradient algorithm then states:

$$
\begin{aligned}
& W_{c d} \leftarrow W_{c d}-\nu_{c d} \frac{\partial}{\partial W_{c d}} D(I, W H), \\
& W_{c d} \leftarrow W_{c d}-\nu_{c d}\left[\sum_{j=1}^{n} I_{c j} \frac{H_{d j}}{\sum_{k=1}^{q} W_{c k} H_{k j}}-\sum_{j=1}^{n} H_{d j}\right] .
\end{aligned}
$$


Forceing the step size

$$
\nu_{c d}=\frac{W_{c d}}{\sum_{j=1}^{n} H_{d j}}
$$

gives

$$
W_{c d} \leftarrow W_{c d} \frac{\sum_{j=1}^{n}\left(H_{d j} I_{c j}\right) / \sum_{k=1}^{q} W_{c k} H_{k j}}{\sum_{j=1}^{n} H_{d j}} .
$$

The iterative computation will stop until the objective function converges. The detailed description of NMF algorithm can be found in (Pascual-Montano et al., 2006; Berrya et al., 2006). The computational procedure is given as follows:

1. Initialize $W$ and $H$ with positive random numbers.

2. For each basis vector $W_{a} \in R^{m \times 1}$, update the corresponding encoding vector $H_{a} \in R^{1 \times n}$, followed by updating and normalizing the basis vector $W_{a}$. Repeat this process until convergence.

Formally, the iteration algorithm in step 2 is as follows:

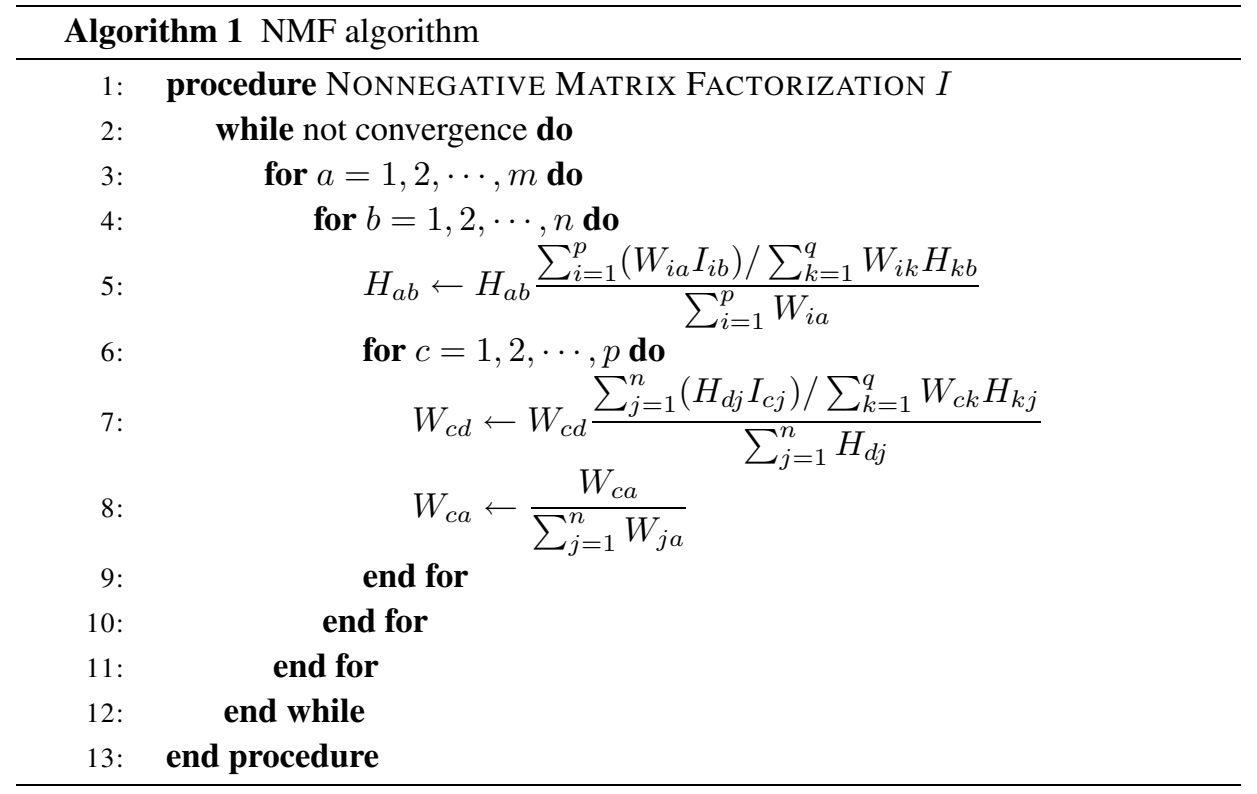

\section{Watermarking Procedure}

In this section, we introduce a novel image watermarking scheme using subsampling and NMF. The detail algorithm is as follows. 


\subsection{Watermark Embedding}

In our proposed scheme, the watermark is a pseudo-random Gaussian sequence $M$ with length $l$, i.e., $M=w_{1} w_{2} \cdots w_{l}$, which is embedded in a given image $I$. An illustration for the watermark embedding process is shown in Fig. 2. Firstly, the image $I$ is decomposed using subsampling equation (1) into $m n$ subimages, i.e., $I_{1}, I_{2}, \cdots, I_{m n}$. For each subimage $I_{i}$ with size $M / m \times N / n$, it is spread to a column vector in a zigzag order:

$$
\begin{aligned}
& I_{i}^{c}(1)=I_{i}(1,1), \\
& I_{i}^{c}(2)=I_{i}(1,2), \\
& I_{i}^{c}(3)=I_{i}(2,1), \\
& I_{i}^{c}(4)=I_{i}(3,1), \\
& I_{i}^{c}(5)=I_{i}(2,2), \\
& \cdots \cdots \ldots \\
& I_{i}^{c}(M N /(m n))=I_{i}(M / m, N / n) .
\end{aligned}
$$

Thus, we obtain $m n$ column vectors $I_{i}^{c}, i=1,2, \cdots, m \times n$. Then, these column vectors are combined into a new matrix $C=\left[I_{1}^{c}, I_{2}^{c}, \cdots, I_{m n}^{c}\right]$ with size $((M N) /(m n)) \times(m n)$. Fig. 3 shows a transposed matrix $C$ from Lenna image, we can see that the column vector in $C$ is very similar and highly correlated, which is natural to be decomposed using NMF, since NMF is originally designed to decompose column-similar matrices. Thus, we used NMF to decompose the matrix $C$, i.e., $C \approx W H$ and produce two nonnegative matrix $W$ and $H$, where $W$ is the normalized basis column vectors and $H$ is the encoding coefficient vectors. Here, the matrix $H$ involves the main local features under the basis matrix $W$, and these features are marked as larger encoding coefficients.

In the proposed scheme, the watermark $M$ is embedded into the $l$ maximum elements of the matrix $H$ randomly. Suppose the watermark element $w_{k}$ is embedded in the coef-

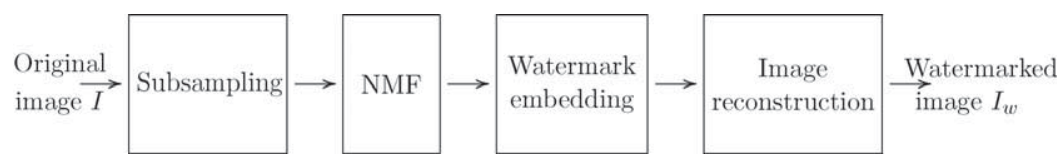

Fig. 2. Watermark embedding process.

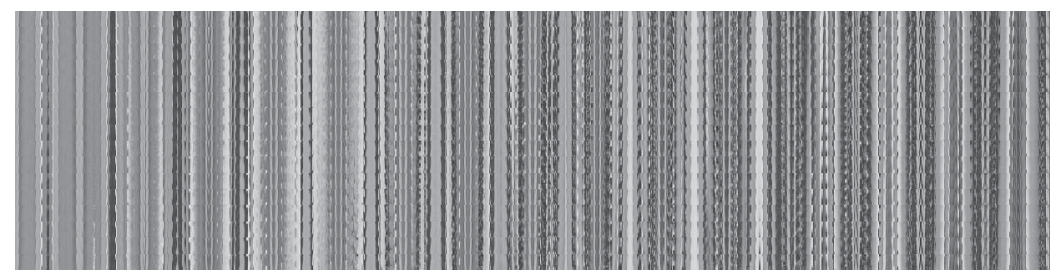

Fig. 3. The transposed matrix $C$ constructed from the subimages, where the subsampling parameter $m, n=16$. 
ficient $H_{i j}$, the embedding algorithm is

$$
H_{i j} \leftarrow H_{i j}+\alpha w_{k}
$$

where, $\alpha$ denotes the watermark embedding strength. Once all the watermark elements are embedded, the new matrix $C$ are constructed using $C \leftarrow W H$, then through image reconstruction and inverse subsampling, the watermarked image $I_{w}$ is obtained. In the watermark embedding process, due to good capability of local decomposition for nonnegative matrix factorization, the maximum elements in the matrix $H$ denotes the local most distinct features under the basis matrix $W$. Thus, the scheme can achieve a better local watermark embedding algorithm, and the robustness will be demonstrated in the following experiments.

\subsection{Watermark Detection}

Given a suspected watermarked image $I^{*}$, for the watermark detection, firstly it is decomposed using subsampling into subimages, the subimages are spread into column vectors as that in the watermark embedding process, and the vectors are combined into a matrix $C^{*}$, then NMF is applied to $C^{*}$, i.e., $C^{*} \approx W^{*} H^{*}$. Suppose the watermark $w_{k}$ is suspected to embedded in the matrix element $H_{i j}^{*}$, we use the following similarity detection to make a decision on whether the image is watermarked as follows:

$$
\rho=\sum_{k=1}^{l} w_{k} H_{i j}^{*} .
$$

Then given a detection threshold $T$, if $\rho \geqslant T$, we think the image $I^{*}$ is watermarked using $M$, otherwise not.

\section{Experiments}

In this section, some experiments are carried out to evaluate the performance of the proposed scheme. Firstly, we test the proposed scheme using the popular test image, Lenna, shown in Fig. 4(a). In the watermarking process, the subsampling parameters $m, n=16$ and $l=1000$, and the watermark embedding strength $\alpha=0.03$. Fig. 4(b) shows the watermarked image, and its watermark detection value $\rho=31.1$. We also used other 1000 random watermark seeds to test the detection process, and the histogram of the detection value is shown in Fig. 5, where only one is the correct watermark. If we set the detection threshold $T=10$, only the correct watermark can be detected.

We tested the robustness of the proposed watermarking scheme using some signal processing distortions, and also we compared the performance of the proposed scheme with the classical DCT based spread spectrum watermarking scheme proposed in (Cox et al., 1997). Fig. 6 shows some experimental results under JPEG compression, Gaussian lowpass filtering and noise addition with different PSNR, which are obtained through 


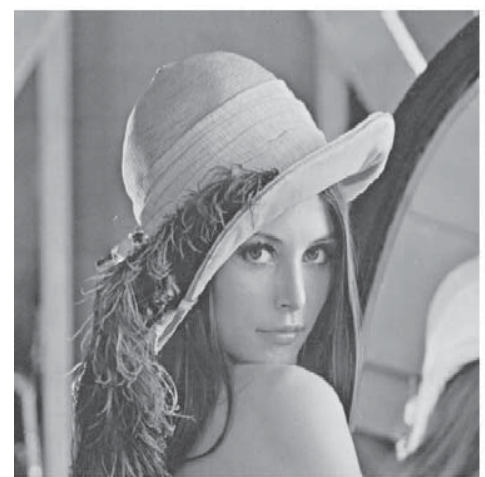

(a)

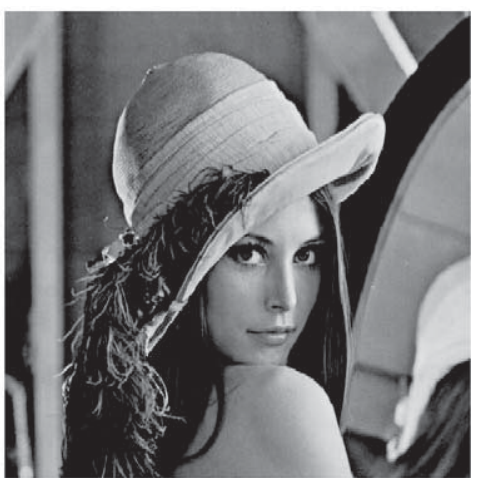

(b)

Fig. 4. (a) The original image, Lenna. (b) A watermarked version of (a).

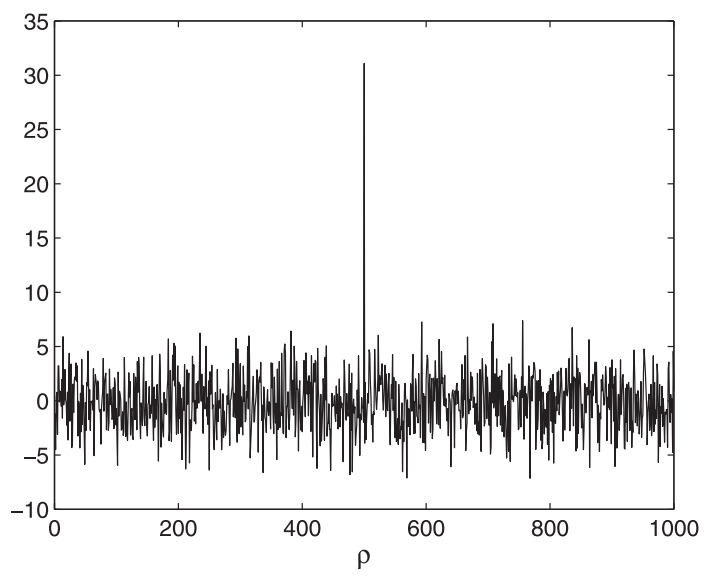

Fig. 5. The response of 1000 watermark seeds, where only one is the correct watermark.

setting different filter parameters and noise strength using Matlab. We can see that the proposed scheme achieves a strong robustness to common signal processing, furthermore, compared with Cox's classical scheme, it is also more robust. Furthermore, Stirmark is also used to test the scheme using some geometrical and other distortions. Table 1 shows some robustness test results under rotation, cropping and resizing, which also show better robustness than Cox's scheme. Especially, we can see that if the detection threshold $T$ is set to 10 , the watermark can still be detection under cropping with better robustness, while Cox's scheme fails.

In order to further evaluate the performance of the proposed scheme, we also build an image database including 1000 test images. Firstly, we used the database to test the robustness under JPEG compression. Fig. 7(a) shows the histogram of the detection value $\rho$ under JPEG quality 60 , which shows that if the threshold $T$ is set to 10 , all of the images can be thought to be watermarked. Then, we test the robustness under geometrical 


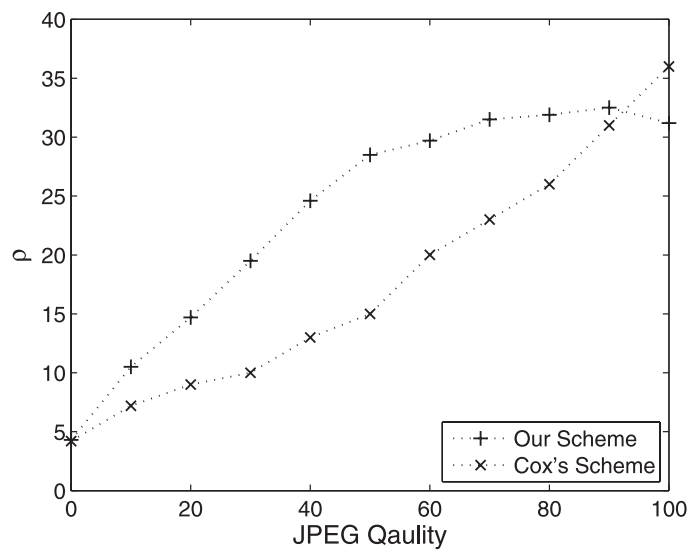

(a)

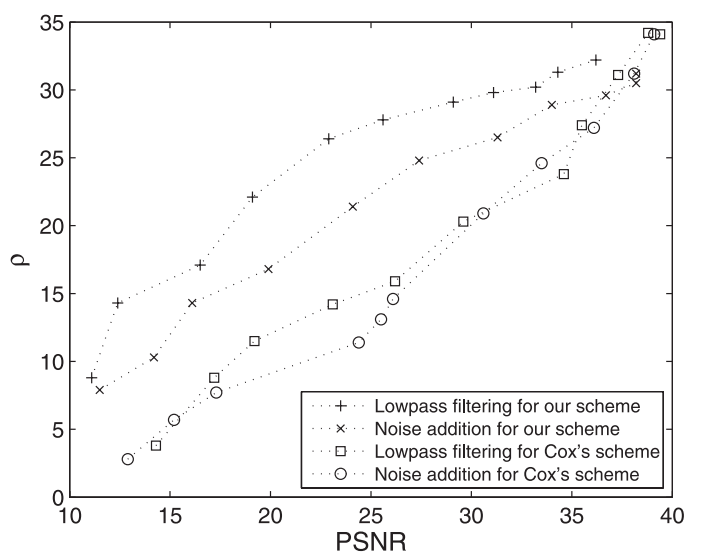

(b)

Fig. 6. Robustness under signal processing. (a) JPEG compression. (b) Gaussian lowpass filtering and noising.

Table 1

Robustness experiments under geometric attacks for the proposed scheme and Cox's scheme

\begin{tabular}{lcc}
\hline \multirow{2}{*}{ Attacks } & \multicolumn{2}{c}{$\rho$} \\
\cline { 2 - 3 } & Our Scheme & Cox's Scheme \\
\hline cropping (10\%) & 21.5 & 8.4 \\
cropping (20\%) & 18.8 & 9.1 \\
cropping (30\%) & 16.9 & 6.3 \\
Resizing (1.2) & 27.7 & 21.9 \\
Resizing $(0.8)$ & 28.8 & 20.2 \\
Rotation $\left(-60^{\circ}\right)$ & 33.3 & 28.1 \\
Rotation $\left(40^{\circ}\right)$ & 33.8 & 27.6 \\
\hline
\end{tabular}




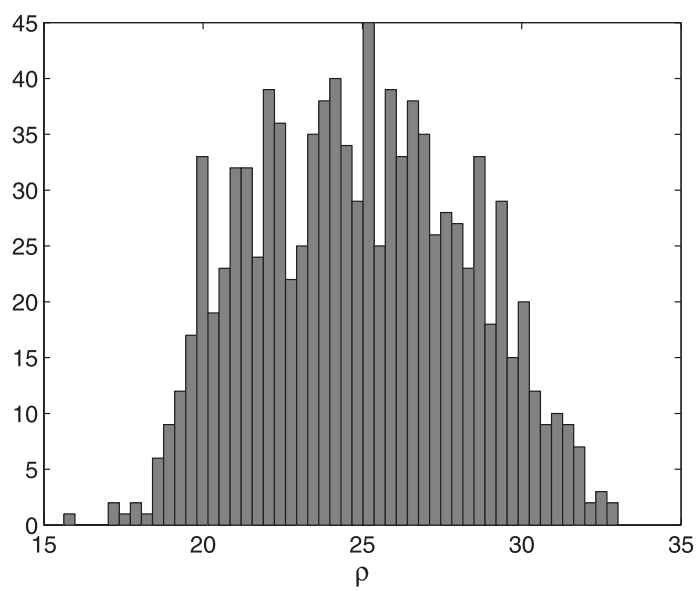

(a)

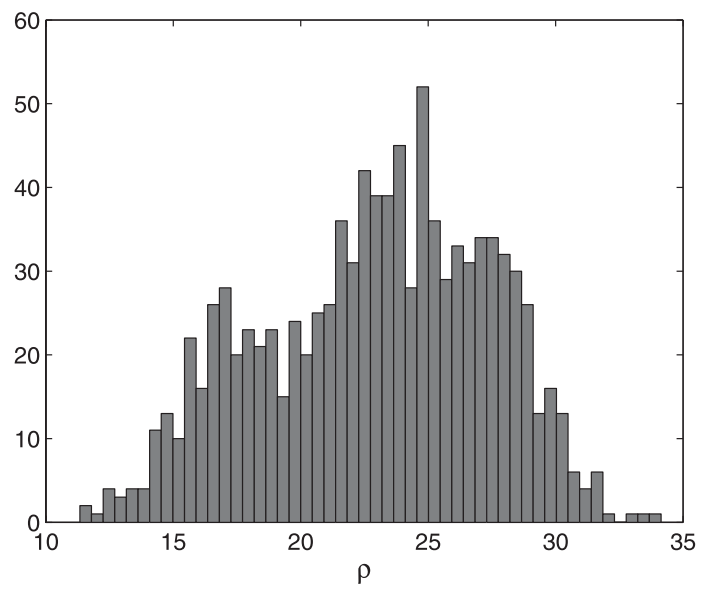

(b)

Fig. 7. The histogram of the detection value $\rho$. (a) 1000 test images under JPEG quality 60. (b) 1000 test images under Gaussian lowpass filtering.

cropping, the percentage for cropping is from $10 \%$ to $30 \%$. Fig. 7(b) shows the histogram of the detection value under cropping, where, if the threshold is set to 10 , over $98 \%$ of the images can be certificated for the corrected watermark, which also shows a good robustness. Furthermore, we also test many other performance with the database, including noising, lowpass filtering, rotation, resizing, etc. all of the experiments show that the proposed watermarking scheme is quite robust to theses attacks.

Finally, we also test the robustness under permutation attack. In (Lu et al., 2005), we proposed a simple permutation attacks, which fail most of current subsampling based watermarking schemes through random permutating the corresponding elements in every subimages. Table 2 gives some robustness test results under common permutation attacks, which shows strong robustness to the permutation attack. 
Table 2

Experimental results for common permutation attacks (Lu et al., 2005)

\begin{tabular}{llllll}
\hline \multirow{2}{*}{ Test image } & \multicolumn{2}{c}{ Watermarked image } & & \multicolumn{2}{c}{ Attacked image } \\
\cline { 2 - 3 } \cline { 6 - 6 } & PSNR & sim & & $P S N R$ & sim \\
\hline Lenna & 37.1857 & 31.1 & & 36.2732 & 30.2 \\
Baboon & 34.2104 & 33.2 & & 32.4481 & 33.5 \\
Barbara & 35.7693 & 29.4 & & 33.4378 & 28.6 \\
\hline
\end{tabular}

\section{Conclusions}

In this paper, we have proposed a novel image watermarking based on subsampling and nonnegative matrix factorization. In the scheme, a pseudo-random Gaussian watermark sequence is embedded in the encoding vectors of NMF in subsampling domain. Experimental results shows that the proposed scheme achieve strong robustness to many signal processing and distortions due to the subimage construction for subsampling and physically meaningful factorization of NMF.

\section{References}

Berrya, M.W., M. Brownea, A.N. Langvilleb, V.P. Paucac and R.J. Plemmons (2006). Algorithms and applications for approximate nonnegative matrix factorization. Computational Statistics \& Data Analysis.

Chang, C.-C., and H.-W. Tseng (2004). VQ-based image watermarking using anti-gray coding. Informatica, 15(2), 147-160.

Chang, C.-S., and C.-C. Chang (2007). A survey of information hiding schemes for digital images. Int. J. Computer Sciences and Engineering Systems, 1(3), 187-200.

Chu, W.C. (2003). DCT-based image watermarking using subsampling. IEEE Trans. Multimedia, 5, 34-38.

Cichocki, A., R. Zdunek and S. ichi Amari (2006). New algorithms for non-negative matrix factorization in applications to blind source separation. In Proc. 2006 IEEE Int. Conf. Acoustics, Speech and Signal Processing, vol. 5. pp. V-621-V-624.

Cox, I.J., J. Killian, T. Leighton and T. Shamoon (1997). Secure spread spectrum watermarking for multimedia. IEEE Trans. Image Processing, 6, 1673-1687.

Ghaderpanah, M., and A.B. Hamza (2006). Secure copyright protection of digital images using nonnegative matrix factorization. In 23rd Biennial Symposium on Communications. pp. 344-347.

Guillamet, D., J. Vitria and B. Schiele (2003). Introducing a weighted non-negative matrix factorization for image classification. Pattern Recognition Letters, 24, 2447-2454.

Hassanien, A.E. (2007). A copyright protection using watermarking algorithm. Informatica, 17(2), 187-198.

Lee, D.D., and H.S. Seung (1999). Learning the parts of objects by non-negative matrix factorization. Nature, 401, 788-791.

Lee, D.D., and H.S. Seung (2001). Algorithms for non-negative matrix factorization. Adv. Neural Inform. Process. Systems, 13, 556-562.

Lo, C.-C., P.-T. Wang, J.-S. Pan and B.-Y. Liao (2007). A more robust subsampling-based image watermarking. IEICE Trans Info. and Systems, E90-D(5), 877-878.

Lu, W., H. Lu and F.-L. Chung (2005). Attacking subsampling-based watermarking. IEICE Trans. Fundamentals, E88-A(11), 3239-3240.

Lu, W., H. Lu and F.-L. Chung (2006). Robust digital image watermarking based on subsampling. Applied Mathematics and Computation, 181(2), 886-893. 
Pascual-Montano, A., J. Carazo, K. Kochi, D. Lehmann and R. Pascual-Marqui (2006). Nonsmooth nonnegative matrix factorization (nsNMF). IEEE Trans. Pattern Analysis and Machine Intelligence, 28(3), 403-415. Zafeiriou, S., A. Tefas, I. Buciu and I. Pitas (2006). Exploiting discriminant information in nonnegative matrix factorization with application to frontal face verification. IEEE Trans. Neural Networks, 17(3), 683-695.

W. Lu received his BSc degree in automation from Northeast University, China in 2002 and his MSc and PhD degrees in computer science from Shanghai Jiao Tong University, China in 2005 and 2007 respectively. He joined the Department of Computer Science, Sun Yat-sen University, China in 2007. His current research interests include multimedia forensics, digital watermarking, pattern recognition and image processing.

H. Lu received his PhD degree in electrical engineering from Southeast University, Nanjing, China, in 1997. He is currently a professor with the Department of Computer Science and Engineering, Shanghai Jiao Tong University, China. His research interests include neural networks, chaos and complex networks, image processing and pattern recognition.

\section{Perdiskretizavimu ir neneigiamos matricos faktorizavimu pagristas patikimas vandens ženklụ įterpimas}

Wei LU, Hongtao LU

Šis straipsnis siūlo naują patikimą schemą, naudojančią perdiskretizavimą ir neneigiamos matricos faktorizavimą, vandens ženklams skaitmeniniame paveiksle ịterpti. Pirmiausia atliekamas perdiskretizavimas paveikslo dalinei sekai gauti. Tada remiantis stulpeliu panašumu, seka išskaidoma faktorizuojant neneigiamą matricą. Gausinè pseudo-atsitiktinè vandens ženklų seka yra įterpiama ị faktorizuotus išskaidymo koeficientus. Dẻl didelio daliniu paveikslu panašumo ir prasmingo faktorizavimo pasiūlyta schema yra patikima, ypač prieš dažnai naudojamą perstatymu ataką. Skaičiuojamasis eksperimentas demonstruoja pasiūlytos schemos gerumą. 